aim of the gynecologist should be to restore, rather than destroy diseased organs. It is not infrequently found that pain in the ovarian region and its attendant reflex phenomena is due to bands of adhesion between the ovary and loops of the intestine. The peristaltic action of the latter keeps the organ in a continuous state of congestion and irritation. Separation of these bands relieves the pain and discomfort, curing the patient as effectually as would the removal of the ovaries. We have a similar train of symptoms induced when the uterus is retroverted and the ovaries bound down by pelvic adhesions. Many of these cases do not require laparotomy in order to bring about a cure. Several have come to the writer's notice where he has been able, by passing two fingers in the rectum and pressing up the cervix, with the thumb in the vagina, to tear off the rectum from the uterus and set free the appendages. It is true that this plan of procedure is only applicable where the adhesions are of limited firmness. In extensive and firm adhesions the danger of laceration of the tube or the ovarian structure would be too great.

Oöphorraphy offers the preferable method of dealing with ovarian displacement, unless caused by marked enlargement from chronic disease.

The inflammatory adhesions, the complete destruction of function, and the reflex phenomena arising from the diseased condition, make removal of the appendages advisable in the majority of cases of hydro- and hæmatosalpinx.

No one can practice abdominal surgery without appreciation of the great boon the introduction of this operation has been to suffering woman, and we cannot do better, in conclusion, than to urge :

I. That the operation for the removal of the appendages should be promptly performed in every case in which it is evident that relief can not be otherwise secured.

2. It should be considered as a dernier ressort where there is a hopeful prospect of restoration to health by less dangerous methods, or without the sacrifice of the reproductive function.

3. Its consideration should be dismissed in every case capable of restoration to health by other plans of treatment.

I8I8 Arch street.

\section{THE USE OF MEN'THOL IN DISEASES OF THE UPPER AIR-PASSAGES.} Read in the Section of Laryngology and Otology, at the Fortieth An
nual Meeting of the American Medical Association. June, I88g.

BY FRANK HAMILTON POTTER, M.D., BUFFALO, $N$. Y.

LECTURER ON DISEASES OF THE NOSE AND THROAT, MEDICAL DEPARTMENT, NIAGARA UNIVERSITY.

The object of this paper is to present a clinical report on the use of menthol in the diseases of the upper air-passages. The writer's attention was first called to this drug in this connection by some remarks of Rosenberg on a paper by Krause, of Berlin, entitled "The Therapy of Laryngeal Phthisis," read in the Laryngeal Sub-section of the Fifty-ninth Meeting of German Naturalists and Physicians in Berlin, September, 1886. Since that time Rosenberg has contributed several papers on this subject, and other writers also have occasionally considered it. Most of the studies, however, that are on record relate to the action of menthol on laryngeal disease, and that chiefly of tubercular origin or arising in tuberculous patients.

Prompted by the favorable opinions expressed by all writers in regard to the use of the drug in the diseases just mentioned, it occurred to me to use it in the inflammatory diseases as seen in the nose, pharynx and mouth as well as in the larynx. The results obtained with it from the date first alluded to to the present time are here presented. These results will be given in a general way and dry statistical detail will be avoided as much as possible.

In reporting the observations concerning the action of any single remedy there is always considerable danger of claiming more for it than a careful study of the facts will warrant. The enthusiasm of the observer may lead him to attribute to it much that rightfully belongs to the natural reparative processes. If this mistake has been made further observation will, no doubt, correct it. I can only say that no discrimination has been exercised, and that favorable and unfavorable results have been alike noted at the time of observation and carefully reconsidered in making this report.

Your attention is first directed to the way the drug has been used. Combinations with other drugs have been almost entirely avoided. Menthol has been simply dissolved in an oil in strengths varying from I to 40 per cent.; olive oil is preferred by most writers, but I have used one of the fluid vaseline preparations called oleum petrolina. Dr, E. L. Shurley, of Detroit, I believe, was the first to call attention to this preparation. It is almost without odor, unchanged by temperature, and of a consistency easily sprayed by any atomizer with a moderately large orifice to the spray tube. Tubes made on the Sass or Devilbiss patterns, or the hand and office atomizers made by the Davidson Rubber Co., numbered 52, 59 and 65, answer the purpose thoroughly. These last are especially mentioned because great difficulty was experienced in obtaining an atomizer that would spray an oily fluid. Most of those advertised for that purpose fail to meet the requirements, Menthol has also been used by means of vaporization and inhalation, and applied by means of an applicator well wrapped with cotton. No one method has been used to the exclusion of the others, and often all 
of them have been employed with the same patient. Menthol can be dissolved in the oleum petrolina up to a strength of 50 per cent. Those above 25 per cent. have been used by inhalation, those below by direct application. If the solution is too strong it will cause great pain and patients will refuse to have it applied. This is so especially in the nose, and I cannot agree with some writers who report using it there as high as 20 per cent. Without cocaine $I$ have never been able to apply to the nasal mucous membrane more than a $\mathrm{I}$ or 2 per cent. solution, excepting in atrophic conditions, which will be considered by themselves. In the pharynx, the mouth and the larynx much stronger solutions can be used.

When applied in the proper percentage menthol produces a pleasant, cool sensation. It is analgesic to some degree and second applications can be made in increased strength without causing discomfort. It is interesting to notice that when used in full strength on the first application patients differ as to the character of the resulting sensation. Some will describe it as a feeling of intense cold and others as a feeling of great heat. This is another illustration of the fact that extreme degrees of heat and cold cannot be easily distinguished.

Let us now inquire into the results obtained from the use of menthol, and first in the diseases of the nose. In the acute diseases of the nose it does not appear to be of much benefit.

It has been used in the ordinary acute rhinitis and in its various forms, such as the membranous and those occurring in patients with the syphilitic and rheumatic constitutions. The feeling of coolness was grateful many times to the patients, but the drug did not seem to have any controlling influence over these conditions. In rare instances where a simple chronic rhinitis, without hypertrophy or deformity, was seen, treatment with menthol yielded better results. I have seen three such cases where supplementing the ordinary treatment with a weak solution of menthol assisted materially in restoring the patients to health. This statement can be made with some degree of certainty because they had been treated in the ordinary way for some time without progress. After operations upon the nasal passages, especially in those cases where a mild antiseptic is necessary, menthol has rendered good service. The soothing properties of the oil combined with the germicidal qualities of the drug have kept the wounds free from sepsis and thus hastened repair. So far as known it has never produced any toxic symptoms, and can therefore be used freely. Following operations, after the nasal passages have been thoroughly cleaned, the parts have been covered with as strong a solution as can be borne by means of the spray, and the excess then blown away. I think to this practice can be attributed the freedom of the cases which form the basis of this report, from the mild sepsis sometimes seen after operations within the nose. These cases have been singularly free from any symptom of sepsis; and as the method of operation only differs in the use of this drug from that followed by more skilful operators who report some cases where a mild sepsis was observed to follow surgical interference with the nasal passages, it seems that menthol should be credited with this pleasing result.

In the treatment of atrophic rhinitis menthol has a place. It cannot supplant other measures, but can supplement and assist in the relief of this most discouraging affection. It has been tried in the following manner: After cleansing the parts a solution that can just be felt has been thoroughly applied. It is remarkable often how strong a percentage will be borne. I have used it in this disease as high as 35 per cent. without the patient complaining. After a few days, however, the parts will become more sensitive and a weaker solution must be applied. With this returning sensitiveness a corresponding benefit takes place in the patient. The dryness, odor, and the accumulation of hardened mucus masses are partially relieved. Beginning with the strength just mentioned, a gradual decrease has been found necessary until, at the end of several weeks, but a 5 per cent. solution would be tolerated. The condition of the patients has improved step by step with the decrease in the toleration of the drug. Whether this improvement will be permanent or not it is difficult to say, as the cases have been too recent, but the results already observed seem to warrant a thorough study of the action of menthol in this disease. Besides the office applications it was found convenient to fit out patients with an atomizer containing the proper solution, with instructions to spray it into the nose from one to three times daily. This plan will lessen the frequency of the office visits, keep the nasal passages free from odor, and prevent the clinging of masses of inspissated mucus to their walls. All this adds to the comfort of the patients and does not interfere in the least with the employment of other means for the relief of the disease.

In the pharynx and mouth the results with menthol have been very satisfactory, and perhaps more positive than in other parts of the upper air tract. It has been used in acute and chronic inflammations of these organs, especially in the latter, with some success. If the ordinary sore throat is taken in its early stages and the parts brushed with a strong solution, frequently repeated, the attack may often be limited both in intensity and duration. This has been seen repeatedly in patients subject to sore throat. They will appear as soon as an attack begins and ask that their throats be treated in the same way as before. I believe these to be cases where the cause is local rather than constitutional. In those depending 
on some systemic condition this treatment does not as often succeed.

Many cases of chronic inflammation in these organs with most diverse etiology have been treated with menthol. In some of these cases the relief has been marked and permanent, in others partial and ternporary. After careful consideration of the results the following general rule can be formulated: When a chronic inflammation follows an acute attack, with the organs above and below the pharynx free from serious disease, the relief from this treatment has been permanent; associated with chronic nasal or laryngeal disease the relief has been partial and temporary. This conclusion is in keeping with the known relations of the pathological processes of the upper air-passages and the action of drugs upon them. However, it mutst be borne in mind that a certain class of cases are relieved by this remedy, and that therefore it is a valuable addition to the list of our therapeutic agents.

Menthol has not been used in acute inflammation of the larynx, but in subacute and chronic inflammation of that organ it has been tried extensively. The results do not differ in any marked degree from those following its use in the pharynx, and the rule just given is referred to as expressing the conclusions of my observations concerning its action here. The applications to the larynx have been by means of the spray, as it was found that this method was more pleasant to the patients and gave as good satisfaction as when applied directly.

Before concluding this report a few words should be said of the action of menthol on the tubercular lesions. My experience has not been large in these cases, and therefore it is with some hesitation that they are referred to at all; still I have seen a few cases, and the importance of the subject justifies the record of the results even if the number of observations is not large.

Menthol has been used in five cases of laryngeal phthisis, all of them consecutive upon disease of the lungs. 'Two were very far advanced, and only lived two and four months respectively from the date of the first observation. Even in these desperate cases the effect of menthol was marked, and they, perhaps, will best illustrate its local action, as but little improvement took place from any general treatment. The drug was used both by inhalation and by the spray, the strength of the former being 30 per cent. and that of the latter Io to 20 per cent. The inhalations were given frequently for a few minutes at a time; the spray was used from three times a day to once every other day according to circumstances.

My limited experience confirms the observations of Rosenberg, Hyndman, and Beehag.

The hypersecretion is diminished and the larynx becomes cleaner. The ulcers also show the good effect and heal after a time, leaving smooth cicatrices, The infiltrations and hypertrophies are slower in responding, and it is sometimes necessary to apply lactic acid or touch them with the galvano-cautery point, or even to use the curette before they will yield, Following up these methods with the menthol treatment seems to hasten the repair as in the case of the ulcers. As the local condition improves dysphagia is lessened and nutrition promoted. The satisfactory results attending the application of this drug to tubercular lesions can be accounted for not only by its analgesic action and by its power to control superficial inflammations, but also by its influence on the bacillus tuberculosis. The investigations of Koch, Sormain and Bagnatelli show that menthol is highly destructive of this bacillus, and to this fact is due, no doubt, the benefit resulting from its use.

In conclusion we may say that while menthol seems to be a drug with a future of great usefulness, it needs to be carefully studied and investigated, so that we may become familiar with its action and know its limitations.

273 Franklin St.

Dr. Jonathan Wright, of Brooklyn, spoke of Heryng's method of curetting laryngeal ulcers and applications of lactic acid. He had seen very good results from the combined use of curetting and lactic acid, more particularly in causing subsidence of subjective symptoms, but he has also seen healing of the ulcers under its use. If we only gain relief from the subjective symptoms we have done a great service to humanity, as there is no disease more appalling in the sufferings it inflicts on its victims.

\section{THE CLINIC.}

\section{THE TREATMEN'T OF LOCOMOTOR} ATAXIA BY SUSPENSION, E'TC.

A Clinical Lecture Deliver at the City Hospital, St. Louts, Mo., December 21,1889 .

BY ALEXANDER B. SHAW, M.D.,

PROFESSOR OF DISEASES OF THE MIND AND NERVOUS SYSTEM HEAUMONT MEDICAL COLLEGE.

[Reported for THE JOURNAL.]

Now, gentlemen, we come to the consideration of the treatment of tabes dorsalis, posterior spinal sclerosis, or locomotor ataxia, as you may be pleased to term it. For this man, who is suffering from that advanced form of the disease known as "Charcot's disease," of course we can do nothing but make the remainder of his days as comfortable as possible; but if the patient be seen during the early stages of the disease, when the lancinating pains are the most prominent symptoms, great benefit will frequently follow the administration of the 